\title{
Cardiac Sympathetic Denervation Can Predict the Wearing- off Phenomenon in Patients with Parkinson Disease
}

\author{
Jee-Eun Lee ${ }^{1}$, Joong-Seok Kim ${ }^{1}$, Dong-Woo Ryu ${ }^{1}$, Yoon-Sang $\mathrm{Oh}^{1}$, Ie Ryung Yoo $^{2}$, and Kwang-Soo Lee ${ }^{1}$ \\ ${ }^{I}$ Department of Neurology, College of Medicine, The Catholic University of Korea, Seoul, Republic of Korea; and ${ }^{2}$ Department of \\ Nuclear Medicine, College of Medicine, The Catholic University of Korea, Seoul, Republic of Korea
}

\begin{abstract}
Recent studies have suggested that preserved cardiac sympathetic denervation may be associated with a small motor burden in Parkinson disease (PD) and serve as a good marker, which is not associated with other nonmotor symptoms. We sought to investigate whether cardiac sympathetic denervation increases the risk of the early wearing-off phenomenon in PD. Methods: This hospitalbased prospective study enrolled 266 de novo patients with PD who underwent ${ }^{123} \mathrm{I}$-metaiodobenzylguanidine ( ${ }^{123} \mathrm{I}-\mathrm{MIBG}$ ) scintigraphy on initial evaluation. The patients visited the outpatient clinic every 2-6 mo and were followed for a minimum of 18 mo from the time they began taking dopaminergic medication. Each patient was assessed for the wearing-off phenomenon on the basis of the clinical assessments and symptom diaries. Clinical events were analyzed from the date of evaluation by ${ }^{123}$ |-MIBG scintigraphy until the date of the first occurrence of the wearing-off phenomenon, or until the last follow-up date without wearing-off. Results: During a mean follow-up period of $30.4 \pm 14.8 \mathrm{mo}, 71$ patients developed wearingoff. The wearing-off phenomenon occurred more in patients with decreased ${ }^{123}$ I-MIBG uptake. A Cox regression analysis revealed that both low ${ }^{123}$ I-MIBG uptake and early onset age significantly predicted the development of wearing-off. Conclusion: Our study suggests that a reduction in myocardial ${ }^{123}$ |-MIBG uptake in PD patients may be associated with a subsequent increased risk for the wearing-off phenomenon. Findings strongly support that PD patients with normal cardiac sympathetic innervation might have less involvement of the midbrain dopaminergic circuitry and a concomitant reduced risk for motor complications, such as wearing-off.
\end{abstract}

Key Words: Parkinson's disease; wearing-off phenomenon; cardiac sympathetic denervation; ${ }^{123}$ I-metaiodobenzylguanidine (MIBG) scintigraphy

J Nucl Med 2018; 59:1728-1733

DOI: 10.2967/jnumed.118.208686

\section{$\mathbf{M}$}

etaiodobenzylguanidine labeled with ${ }^{123}$ I ( $\left.{ }^{123} \mathrm{I}-\mathrm{MIBG}\right)$ is a physiologic noradrenaline analog that plays a role as a tracer for the uptake, transport, and subsequent vesicular storage of noradrenaline in presynaptic sympathetic nerve terminals (1). Postganglionic presynaptic cardiac sympathetic nerve endings can be

Received Jan. 21, 2018; revision accepted Mar. 5, 2018.

For correspondence or reprints contact: Joong-Seok Kim, Department of Neurology, College of Medicine, The Catholic University of Korea, Seoul St. Mary's Hospital, 222, Banpo-daero, Seocho-gu, Seoul, 06591 Republic of Korea.

E-mail: neuronet@catholic.ac.kr

Published online Mar. 23, 2018.

COPYRIGHT (C) 2018 by the Society of Nuclear Medicine and Molecular Imaging. noninvasively assessed by ${ }^{123}$ I-MIBG scintigraphy, so this technique has been used to support clinical diagnoses for both cardiac and various neurologic diseases (2). Myocardial ${ }^{123}$ I-MIBG uptake is reduced in patients with Lewy body diseases such as Parkinson disease (PD), as well as dementia with Lewy bodies (3). Many studies have demonstrated that myocardial ${ }^{123}$ I-MIBG scintigraphy can be useful for the early differential diagnosis of patients with PD from other atypical PD, such as multiple-system atrophy, as a decrease in ${ }^{123}$ I-MIBG uptake often exists even in the early stages of PD $(2,4,5)$.

Some studies have indicated that the onset age and the disease duration, severity, and specific phenotype influence ${ }^{123}$ I-MIBG uptake in PD, and others have suggested that patients with a mild Hoehn and Yahr (H\&Y) stage, short disease duration, slow progression of motor dysfunction, and preserved cognitive function may have fewer myocardial dysfunctions (6-9). We recently reported that patients with a normal ${ }^{123} \mathrm{I}-\mathrm{MIBG}$ scan have a relative lower prevalence of nonmotor symptoms than those with decreased ${ }^{123} \mathrm{I}$ MIBG uptake, suggesting that normal cardiac sympathetic innervation in PD is associated with a low pathologic burden in the heart (10). However, there have been few studies about using ${ }^{123} \mathrm{I}-\mathrm{MIBG}$ scintigraphy for prognosis prediction (11).

The hypothesis tested in this study was that cardiac sympathetic denervation as one of measures of peripheral $\alpha$-synuclein may contribute to the early motor wearing-off phenomenon as one of markers of PD progression. In this study, we analyzed the predictive value of ${ }^{123}$ I-MIBG scintigraphy with regards to the occurrence of wearingoff in de novo PD patients.

\section{MATERIALS AND METHODS}

\section{Patients and Clinical Assessments}

The Institutional Review Board of Seoul St. Mary's Hospital, of the Catholic University of Korea, Seoul, approved the study protocol, and all subjects provided written informed consent to participate.

A total of 266 patients at the Movement Disorder Clinic, Department of Neurology at Seoul St. Mary's Hospital between January 2010 and September 2015 were diagnosed with PD according to the U.K. Brain Bank criteria (12): the presence of appropriate dopamine transporter defects on dopamine transporter imaging scans and the presence of PD drug response during follow-up. These PD patients were followed for a minimum of 18 mo from the time they began taking dopaminergic medication. Some of cohorts enrolled in this prospective study included all patients' previously published research (10). The age-matched controls (mean age $\pm \mathrm{SD}, 67.4 \pm 4.8 \mathrm{y}$ ) were recruited from the community with no evidences of neurologic and psychiatric disorders.

Clinical information, including age, age at the symptomatic disease onset, sex, disease duration, and history of arterial hypertension and 
diabetes mellitus, was collected. All patients underwent MRI. All patients were evaluated using the Unified Parkinson Disease Rating Scale (UPDRS) and the modified H\&Y stage.

The exclusion criteria included neurologic abnormalities related to atypical PD or secondary parkinsonism, abnormalities in the basal ganglia or cerebellum on structural MRI scans, a history of diabetic neuropathy or other peripheral/autonomic neuropathy, a history of relevant cardiac disease or any abnormalities on routine chest radiography or electrocardiography, and taking medications known to influence autonomic functions or those reported to influence myocardial ${ }^{123}$ I-MIBG uptake.

The patients visited the outpatient clinic every 2-6 mo, and were followed for a maximum of $7 \mathrm{y}$ from the time they began taking dopaminergic medication. Their PD pharmacotherapy included levodopa, dopamine agonists, anticholinergics, monoamine oxidase B, and catechol-O-methyl transferase inhibitors, or amantadine. Each patient was assessed for the wearing-off phenomenon on the basis of the clinical assessments that were conducted during each visit and Parkinson symptom diaries. The wearing-off phenomenon was defined as the generally predictable recurrence of motor and nonmotor symptoms that preceded the next scheduled antiparkinsonian medication dosage (13). The wearoff time should also be greater than $1.5 \mathrm{~h}$ per day despite optimization of dopaminergic treatment. In all patients with wearing-off, they were taking more than 3 doses of dopaminergic medication per day. In all enrolled patients, levodopa equivalent daily doses were calculated according to the usual formula (14), and the duration of antiparkinsonian medication was calculated at the endpoint.

\section{3|-MIBG Scintigraphic Methods}

${ }^{123}$ I-MIBG scintigraphy was performed using a dual-head camera equipped with a low-energy, high-resolution collimator (Siemens), and data were collected for 30-min (early) and 2-h (late) time points after injection of $111 \mathrm{MBq}$ of ${ }^{123} \mathrm{I}$-MIBG. A static image was obtained with a $128 \times 128$ matrix. Regions of interest were manually drawn around the heart, mediastinum, and lung. Tracer uptake was measured within each region of interest to calculate the heart-to-mediastinum $(\mathrm{H} / \mathrm{M})$ ratio. Myocardial ${ }^{123} \mathrm{I}-\mathrm{MIBG}$ washout was calculated as follows: $\{($ early $\mathrm{H} / \mathrm{M}-$ late $\mathrm{H} / \mathrm{M}) /$ early $\mathrm{H} / \mathrm{M}\} \times 100(15)$.

\section{Statistical Analysis}

Differences in the distributions of demographics were assessed with Pearson $\chi^{2}$ tests for categoric variables, or by the 2-sample $t$ test for continuous variables.

Patients were stratified to normal and abnormal ${ }^{123} \mathrm{I}-\mathrm{MIBG}$ uptake groups using late $\mathrm{H} / \mathrm{M}$ ratios. Because the $\mathrm{H} / \mathrm{M}$ ratios of age-matched controls were normally distributed $(P=0.200$ by 1 -sample Kolmogorov-Smirnov test), the values within the reference limit of the normal H/M ratio were calculated as lower limits $=$ mean $-\left(t_{0.975,11} \times\right.$ $\sqrt{ }(n+1) / n \times \mathrm{SD})$ of 25 age-matched normal controls (cutoff value $=$ $2.26-2.06 \times 1.02 \times 0.23=1.78)$.

Patients were also divided into 4 groups on the basis of late H/M ratio by quartile distribution, and the odds ratios were calculated for the occurrence of wearing-off phenomenon using binary logistic regression analysis adjusted for age.

We used the Kaplan-Meier product-limit method to estimate the cumulative proportion of patients who experienced the wearing-off phenomenon in the whole cohort and in groups that were stratified according to their demographic and clinical variables. The survival time was calculated from the date of the evaluation using ${ }^{123}$ I-MIBG scintigraphy until the date of the occurrence of the wearing-off phenomenon, or until the last follow-up date without wearing-off. Data at the time of death from causes other than by documented PD or loss of contact with the study subjects (5 patients) during the follow-up period were excluded. Testing of the hypothesis was performed by the log-rank test; the effect of predictive variables is expressed as risk ratios and 95\% confidence intervals (CIs), which were calculated using the Cox proportional

TABLE 1

Cohort Clinical Characteristics

\begin{tabular}{|c|c|c|c|c|}
\hline Variable & Total $(n=266)$ & WO- $(n=195)$ & $\mathrm{WO}+(n=71)$ & $P$ \\
\hline Age at onset $(y)$ & $66.1 \pm 9.5$ & $67.0 \pm 9.3$ & $63.4 \pm 9.6$ & 0.007 \\
\hline Male $(n)$ & $129(48.5 \%)$ & 89 (45.6\%) & $40(56.3 \%)$ & 0.123 \\
\hline Disease duration (mo) & $14.1 \pm 15.3$ & $14.5 \pm 15.9$ & $12.9 \pm 13.5$ & 0.444 \\
\hline Hypertension (n) & $109(41.0 \%)$ & 85 (43.6\%) & $24(33.8 \%)$ & 0.151 \\
\hline Diabetes mellitus $(n)$ & $34(12.8 \%)$ & $27(13.8 \%)$ & 7 (9.9\%) & 0.389 \\
\hline UPDRS total & $25.0 \pm 15.5$ & $25.0 \pm 16.1$ & $25.0 \pm 13.6$ & 0.994 \\
\hline UPDRS part 1 & $2.4 \pm 2.1$ & $2.4 \pm 2.2$ & $2.3 \pm 1.8$ & 0.888 \\
\hline UPDRS part 2 & $7.4 \pm 5.1$ & $7.4 \pm 5.6$ & $7.3 \pm 3.7$ & 0.938 \\
\hline UPDRS part 3 & $15.2 \pm 10.0$ & $15.1 \pm 10.0$ & $15.3 \pm 10.0$ & 0.901 \\
\hline Hoehn and Yahr stage & $1.7 \pm 0.7$ & $1.7 \pm 0.7$ & $1.6 \pm 0.7$ & 0.664 \\
\hline Time to endpoint, mo & $30.2 \pm 14.4$ & $31.4 \pm 14.3$ & $26.8 \pm 14.2$ & 0.022 \\
\hline LEDD (endpoint) & $518.7 \pm 275.9$ & $473.9 \pm 246.2$ & $641.6 \pm 315.0$ & $<0.001$ \\
\hline Early $\mathrm{H} / \mathrm{M}$ & $1.66 \pm 0.38$ & $1.73 \pm 0.40$ & $1.48 \pm 0.24$ & $<0.001$ \\
\hline Late $\mathrm{H} / \mathrm{M}$ & $1.63 \pm 0.47$ & $1.72 \pm 0.49$ & $1.40 \pm 0.29$ & $<0.001$ \\
\hline Washout rate & $2.4 \pm 10.9$ & $2.2 \pm 11.5$ & $3.3 \pm 11.0$ & 0.470 \\
\hline Late $\mathrm{H} / \mathrm{M}$ ratio $\leq 1.78$ & 177 (66.5\%) & $112(57.4 \%)$ & 65 (91.5\%) & $<0.001$ \\
\hline
\end{tabular}

Analyses were performed by an independent sample $t$ test or a $\chi^{2}$ test.

WO = wearing-off; LEDD = levodopa equivalent daily dose.

Values represent mean with SD or number of subjects, with percentages in parentheses. 


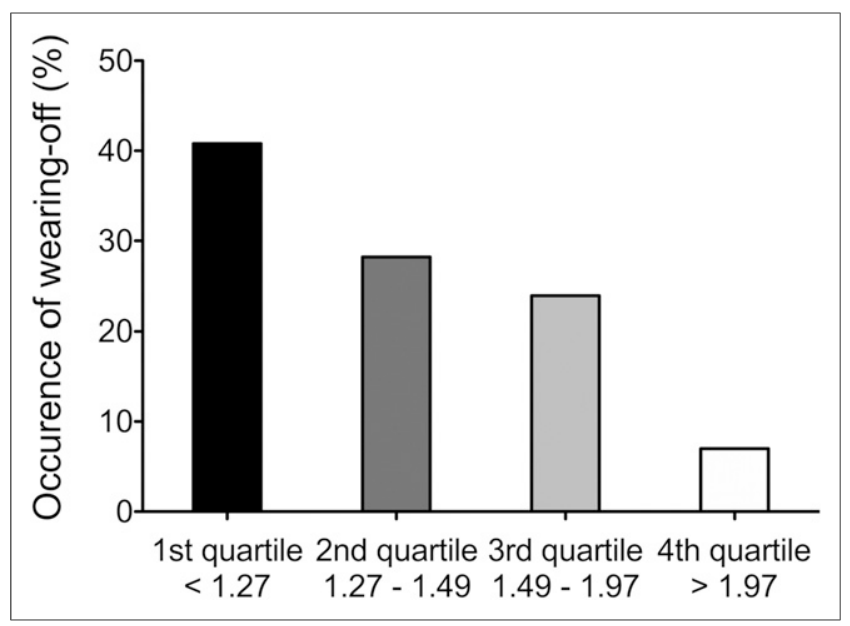

FIGURE 1. Occurrence of wearing-off phenomenon $(n=71)$ in relation to late $\mathrm{H} / \mathrm{M}$ ratio. Peak occurrence was lowest $\mathrm{H} / \mathrm{M}$ quartile $(<1.27)$, with progressive decline for higher $\mathrm{H} / \mathrm{M}$ ratios.

hazards model. The age of onset (early-onset [ $\leq 65 \mathrm{y}]$ vs. late-onset [ $>65 \mathrm{y}]$ ), disease duration ( $\leq 1$ vs. $>1 \mathrm{y}), \mathrm{H} \& \mathrm{Y}$ stage score $(\leq 2$ [no axial involvement] vs. $>2$ [axial involvement]), myocardial ${ }^{123} \mathrm{I}-$ MIBG uptake (normal vs. abnormal/decreased $\mathrm{H} / \mathrm{M}$ ratios), and their combinations were analyzed as categoric variables. A multivariate analysis was performed using the Cox proportional hazards model using those variables associated with risk in the univariate testing protocol. All analyses were performed using SPSS/Mac software, version 24.0. For all tests, the significance level was established at a value of $P$ less than 0.05 .

\section{RESULTS}

From the 337-patient registry, 22 patients with multiple-system atrophy, 21 with progressive supranuclear nuclear palsy, and 23 with dementia with Lewy bodies were excluded in a retrospective manner. Among the remaining 271 PD patients, 5 patients with PD were excluded due to follow-up loss within $1 \mathrm{y}$. Therefore, a total of 266 PD patients were finally enrolled.

The baseline characteristics of patients are summarized in Table 1. Among the 266 patients with PD, 129 were men (48.5\%). The mean age at onset $( \pm \mathrm{SD})$ was $66.1 \pm 9.5 \mathrm{y}$. The mean disease duration was $14.1 \pm 15.3 \mathrm{mo}$. The total UPDRS and H\&Y stage scores were $25.0 \pm 15.5$ and $1.7 \pm 0.7$, respectively. The mean early and late $\mathrm{H} / \mathrm{M}$ ratios were $1.66 \pm 0.38$ and $1.63 \pm 0.47$, respectively. Eightynine patients $(33.5 \%)$ had a normal ${ }^{123}$ I-MIBG uptake, with a late $\mathrm{H} / \mathrm{M}$ ratio $>1.78$. The mean follow-up duration was $30.2 \pm 14.4$ mo, and the mean levodopa equivalent daily dose at the endpoint was $518.7 \pm 275.9 \mathrm{mg}$.

When the patients were divided into 2 groups according to the presence of wearing-off symptoms, the group with wearing-off was younger than those without wearing-off. As expected, the mean time to endpoint was shorter and the mean levodopa equivalent daily dose was higher in the wearing-off group than in the no-wearing-off group. However, each group of patients was similar with regard to disease duration, medical history (hypertension and diabetes), and PD severity (UPDRS and H\&Y stage scores).

TABLE 2

Results of Univariate Test for Wearing-off Prognostic Variables

\begin{tabular}{|c|c|c|c|c|}
\hline Variable & No. of patients & No. of wearing-off events & Cumulative incidence & $P$ \\
\hline \multicolumn{5}{|l|}{ Age at onset (y) } \\
\hline$\leq 65$ & 118 & 43 & $36.4 \%$ & 0.008 \\
\hline$>65$ & 148 & 28 & $18.9 \%$ & \\
\hline \multicolumn{5}{|l|}{ Sex } \\
\hline Male & 129 & 40 & $31.0 \%$ & 0.158 \\
\hline Female & 137 & 31 & $22.6 \%$ & \\
\hline \multicolumn{5}{|l|}{ Disease duration (mo) } \\
\hline$\leq 12$ & 181 & 52 & $28.7 \%$ & 0.199 \\
\hline$>12$ & 85 & 19 & $22.4 \%$ & \\
\hline \multicolumn{5}{|l|}{ Hoehn and Yahr stage } \\
\hline Without postural involvement $(\leq 2)$ & 200 & 57 & $28.5 \%$ & 0.266 \\
\hline Without postural involvement $(>2)$ & 66 & 14 & $21.2 \%$ & \\
\hline \multicolumn{5}{|l|}{ Hypertension } \\
\hline No & 157 & 47 & $29.9 \%$ & 0.089 \\
\hline Yes & 109 & 24 & $22.0 \%$ & \\
\hline \multicolumn{5}{|l|}{ Diabetes mellitus } \\
\hline No & 232 & 64 & $27.6 \%$ & 0.143 \\
\hline Yes & 34 & 7 & $20.6 \%$ & \\
\hline \multicolumn{5}{|l|}{ Late $\mathrm{H} / \mathrm{M}$ ratio } \\
\hline Normal (>1.78) & 89 & 6 & $6.7 \%$ & $<0.001$ \\
\hline Decreased $(\leq 1.78)$ & 177 & 65 & $36.7 \%$ & \\
\hline Total cohort & 266 & 71 & $26.7 \%$ & \\
\hline
\end{tabular}



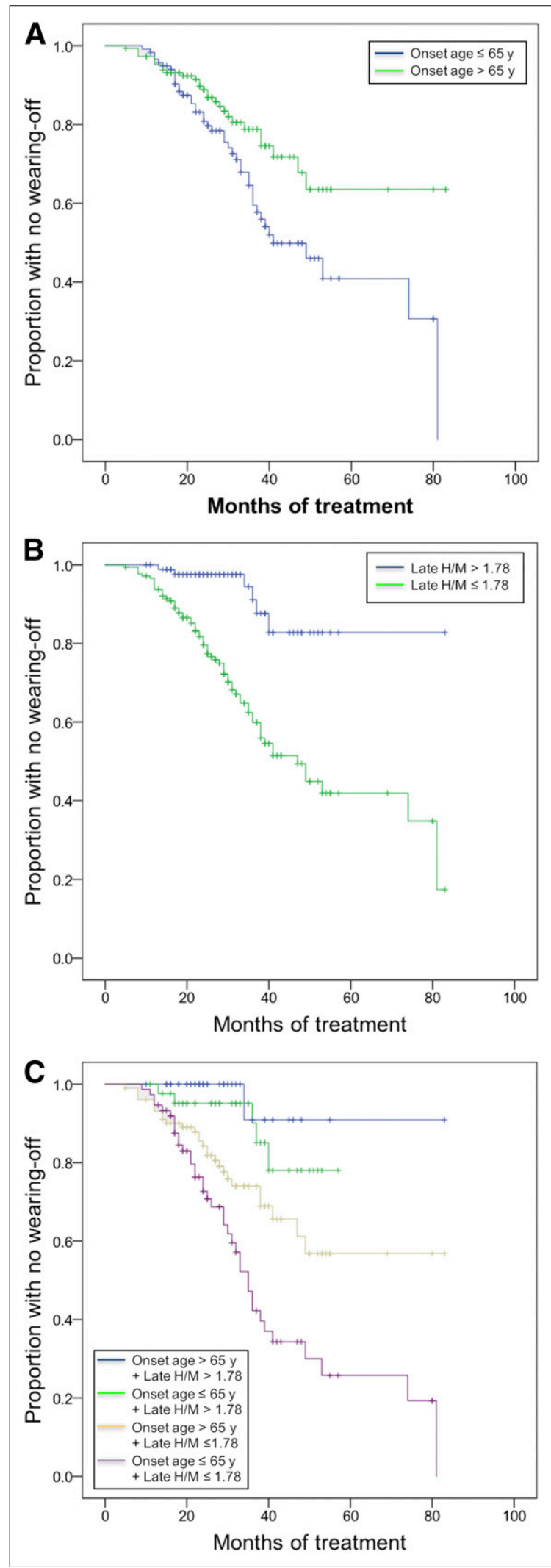

FIGURE 2. Kaplan-Meier estimates of rate of occurrence of wearingoff phenomenon over time. The data are stratified according to the age at onset (A), normal/decreased cardiac sympathetic innervation (B), and their combinations (C). Testing for significance was by log-rank method.
Patients with wearing-off had a higher frequency of decreased $\mathrm{H} / \mathrm{M}$ ratios with a late $\mathrm{H} / \mathrm{M}$ ratio $\leq 1.78$ (wearing-off vs. no wearingoff $=65$ [91.5\%] vs. 112 [57.4\%], respectively; $\chi^{2}=27.206 ; P<$ 0.001 ) and lower $\mathrm{H} / \mathrm{M}$ ratio than those with no wearing-off (Table 1). The occurrence of wearing-off phenomenon in relation to late $\mathrm{H} / \mathrm{M}$ ratio is shown in Figure 1. When compared with patients of the highest late $\mathrm{H} / \mathrm{M}$ quartile $(>1.97)$, patients in the lower 3 quartiles had higher odds for more occurrence of wearing-off. The odds ratios were 4.479 (95\% CI: $1.490-13.462, P=0.008), 6.716$ (95\% CI: 2.248-20.064, $P=0.001$ ) and 14.801 (95\% CI: 4.862-45.064, $P<0.001$ ), when late $\mathrm{H} / \mathrm{M}$ ratio ranged from 1.49 to $1.97,1.27$ to 1.49 , and $<1.27$, respectively.

Seventy-one patients had wearing-off during the follow-up period, yielding an estimated cumulative incidence of $26.7 \%$. Several clinical variables were examined for their effect on the risk of the occurrence of wearing-off (Table 2). The time to the occurrence of wearing-off did not vary significantly with the sex, disease duration, motor symptom severity, or presence or absence of diabetes. The risk was elevated, however, in the patients with younger-onset PD (Fig. 2A; risk ratio: 2.378; 95\% CI: $1.417-$ 3.990). Those with hypertension also had a relatively low risk of wearing-off, with borderline significance (risk ratio: 0.626; $95 \%$ CI: 0.377-1.042) (Table 3).

An abnormal ${ }^{123}$ I-MIBG scan was also a strong predictor of the risk for the occurrence of wearing-off. On Kaplan-Meier survival analysis, among the 71 patients who had wearing-off, the group with decreased ${ }^{123}$ I-MIBG scintigraphy had a 3-y occurrence rate of $36.7 \%$. In contrast, only 6 patients who had normal ${ }^{123}$ I-MIBG scintigraphy also experienced wearing-off, for a 3-y occurrence rate of $6.7 \%$ (Fig. 2B; risk ratio for abnormal ${ }^{123} \mathrm{I}-\mathrm{MIBG}$ : 6.418; 95\% CI: 2.730-15.088) (Table 3).

The combination of younger-onset of disease with decreased ${ }^{123}$ I-MIBG scan had a synergistic effect, with a wearing-off occurrence rate of $50.7 \%$ (Table 4 and Fig. 2C; risk ratio: 21.396; 95\% CI: 2.936-155.938, $P=0.003$ ). The group with older-onset and decreased ${ }^{123}$ I-MIBG scan also had a high risk of wearing-off, compared with those with older-onset and normal ${ }^{123}$ I-MIBG (risk ratio: 10.036 ; $95 \% \mathrm{CI}$ : $1.362-73.933, P=0.024)$.

\section{DISCUSSION}

This study demonstrated that decreased myocardial ${ }^{123}$ I-MIBG uptake increases the risk for the development of the wearing-off phenomenon in PD patients. In addition, the results of this study also showed that a young onset age of PD was associated with a

TABLE 3

Cox Proportional Hazard Ratios for Wearing-Off Phenomenon According to Patient Characteristics

\begin{tabular}{llr}
\hline \multicolumn{1}{c}{ Variable } & Hazard ratio (95\% Cl) & $P$ \\
\hline Age at onset $\leq 65$ y & $2.378(1.417-3.990)$ & 0.001 \\
Male sex & $1.261(0.782-2.034)$ & 0.341 \\
Hypertension & $0.626(0.377-1.042)$ & 0.071 \\
Diabetes mellitus & $0.648(0.284-1.477)$ & 0.302 \\
Disease duration $>12$ mo & $0.953(0.517-1.755)$ & 0.876 \\
Hoehn and Yahr stage $>2$ & $1.267(0.632-2.536)$ & 0.505 \\
Late H/M ratio $\leq 1.78$ & $6.418(2.730-15.088)$ & $<0.001$ \\
\hline
\end{tabular}


TABLE 4

Results of Univariate Test for Combinations of Wearing-off Prognostic Variables

\begin{tabular}{lccc}
\hline Variable & No. of patients & No. of wearing-off events & Cumulative incidence \\
\hline Age at onset $>65 \mathrm{y}+$ late $\mathrm{H} / \mathrm{M}$ ratio $>1.78$ & 46 & 1 & $2.2 \%$ \\
Age at onset $\leq 65 \mathrm{y}+$ late $\mathrm{H} / \mathrm{M}$ ratio $>1.78$ & 43 & 5 & $11.6 \%$ \\
Age at onset $>65 \mathrm{y}+$ late $\mathrm{H} / \mathrm{M}$ ratio $\leq 1.78$ & 102 & 27 & $26.5 \%$ \\
Age at onset $\leq 65 \mathrm{y}+$ late H/M ratio $\leq 1.78$ & 75 & 38 & $50.7 \%$ \\
Total cohort & 266 & 71 & $26.7 \%$ \\
\hline
\end{tabular}

higher risk for the occurrence of wearing-off. The results of our study are consistent with those of a previous Japanese preliminary study with 23 PD patients, which showed a significant difference in the occurrence of wearing-off between the 2 groups that were divided according to $\mathrm{H} / \mathrm{M}$ ratio (11). On the basis of the findings from our study and the previous one, impaired ${ }^{123}$ I-MIBG uptake in PD patients could be an independent factor that can predict the complication of the wearing-off phenomenon.

The wearing-off phenomenon is an important motor complication that occurs after a few years of using dopaminergic treatments; however, the pathophysiology underlying the wearing-off phenomenon is not fully understood. Wearing-off is mainly thought to reflect the progressive degeneration of nigrostriatal dopaminergic neurons, resulting in a reduced storage capacity for levodopa and a subsequent change in the pharmacodynamic response (16).

Recent data have suggested that the cellular pathologies in PD develop in parallel at similar rates, although the first symptoms can often be found in the peripheral nervous system, rather than the midbrain dopaminergic circuitry (17). Likewise, cardiac autonomic dysfunction also appears before obvious motor symptoms in PD patients, and also in $\alpha$-synuclein model transgenic mice (18-20). This is associated with an earlier functional threshold in the autonomic nervous system, and it is hypothesized that neuronal damage in PD occurs in a spreading and ascending pattern from peripheral nerves to the brain stem and midbrain.

Normal ${ }^{123}$ I-MIBG uptake is also associated with less sympathetic denervation and might be associated with less Lewy body pathology in the heart, compared with those with decreased ${ }^{123} \mathrm{I}-$ MIBG uptake. Therefore, we can postulate that PD patients with normal cardiac sympathetic innervation might have less involvement of the midbrain dopaminergic circuitry, and a concomitant reduced risk for motor complications, such as wearing-off. We recently reported the association between orthostatic hypotension, a rapid-eye-movement sleep behavioral disorder, and impaired cardiac sympathetic denervation (10).

Estimates of the incidence of wearing-off vary greatly. Previous studies have demonstrated that approximately $40 \%-50 \%$ of PD patients who were treated with levodopa for 4-6 y experienced wearingoff. The occurrence of wearing-off increases gradually with time, with almost all PD patients developing it within $10 \mathrm{y}$ of initiation of levodopa therapy $(13,21-23)$. Because the wearing-off phenomenon significantly affects the quality of life and can be an important source of disability in PD patients, it is important to identify the occurrence of wearing-off and to start managing it immediately.

Many studies have reported that a young age at the onset of PD is a risk factor for the development of wearing-off and dyskinesia (23-28). In our study, the incidence of wearing-off in the olderonset patient group $(>65 \mathrm{y})$ was significantly lower than that in the younger-onset patient group. The results of this study provide further evidence that a young age at the onset of PD is one of the strong predictive factors for the occurrence of wearing-off.

This study has several limitations. First, there is currently no universal definition of the wearing-off phenomenon. A wide range of motor and nonmotor features are related to wearing-off in PD, and therefore estimates of this phenomenon can vary greatly depending on the study design and definition used. In this study, we used the recently provided consensus definition by an international group of movement disorder specialists (13). Second, this study showed that the risk of wearing-off in male PD patients was mildly elevated compared with that of female patients. Several previous studies, however, revealed that female sex was associated with an increased risk of developing wearing-off $(23,25,29)$. These studies reported that lower body weight; higher daily levodopa doses adjusted to body weight; greater levodopa bioavailability; effects of nondopaminergic therapies for nonmotor symptoms, such as anxiety, mood change, and pain; levels of the female sex hormone (estrogen); and uric acid levels in PD female patients may influence the occurrence of wearing-off, as well as the rate of PD progression (29-31). Our study did not match these correlations. In our study population, male PD patients were mildly younger than female patients (male vs. female $=65.3 \pm 9.7$ vs. $66.8 \pm 9.3 \mathrm{y}$, respectively; $P=0.212$ ), and this may be associated with the inconsistencies between our results and prior studies. We can postulate the physical aging effect on wearing-off is greater than sex effect. In addition, we did not evaluate other factors that may influence the occurrence of wearing-off. Several risk factors for the development of wearing-off have been reported, including a lower body weight, higher disease severity, higher levodopa dose, longer duration of levodopa therapy, treatment with entacapone, and high scores on the UPDRS parts II and III and North American geographic region $(23,25,27,28)$.

\section{CONCLUSION}

This study showed that a reduction in myocardial ${ }^{123}$ I-MIBG uptake in PD patients may be associated with a subsequent increased risk in the occurrence of the wearing-off phenomenon. The results of this study suggest that screening for cardiac sympathetic denervation can identify a subgroup of patients with PD who will experience a more rapid progression than average PD patients.

\section{DISCLOSURE}

This research was supported by Basic Science Research Program through the National Research Foundation of Korea (NRF) funded by the Ministry of Science, ICT and Future Planning (NRF2017R1D1A1B06028086). Prof. Joong-Seok Kim has received 
grants from the National Research Foundation of Korea (NRF) funded by the Ministry of Science, ICT, and Future Planning and Korean Movement Disorders Society (KMDS). No other potential conflict of interest relevant to this article was reported.

\section{REFERENCES}

1. Wieland DM, Wu J, Brown LE, Mangner TJ, Swanson DP, Beierwaltes WH. Radiochemistry and radiopharmaceuticals: radiolabeled adrenergic neuron-blocking agents: adrenomedullary imaging with I-131 iodobenzylguanidine. J Nucl Med. 1980;21:349-353.

2. Nagayama H, Hamamoto M, Ueda M, Nagashima J, Katayama Y. Reliability of MIBG myocardial scintigraphy in the diagnosis of Parkinson's disease. J Neurol Neurosurg Psychiatry. 2005;76:249-251.

3. Kim JS, Park HE, Oh YS, et al. ${ }^{123}$ I-MIBG myocardial scintigraphy and neurocirculatory abnormalities in patients with dementia with Lewy bodies and Alzheimer's disease. J Neurol Sci. 2015;357:173-177.

4. Yoshita M. Differentiation of idiopathic Parkinson's disease from striatonigral degeneration and progressive supranuclear palsy using iodine-123 meta-iodobenzylguanidine myocardial scintigraphy. J Neurol Sci. 1998;155:60-67.

5. Orimo S, Suzuki M, Inaba A, Mizusawa H. ${ }^{123}$ I-MIBG myocardial scintigraphy for differentiating Parkinson's disease from other neurodegenerative parkinsonism: a systematic review and meta-analysis. Parkinsonism Relat Disord. 2012;18:494-500.

6. Hamada K, Hirayama M, Watanabe H, Kobayashi R, Ito H, Ieda T. Onset age and severity of motor impairment are associated with reduction of myocardial ${ }^{123} \mathrm{I}-\mathrm{MIBG}$ uptake in Parkinson's disease. J Neurol Neurosurg Psychiatry. 2003;74:423-426.

7. Spiegel J, Hellwig D, Farmakis G, Jost WH, Samnick S, Fassbender K. Myocardial sympathetic degeneration correlates with clinical phenotype of Parkinson's disease. Mov Disord. 2007;22:1004-1008.

8. Sakakibara R, Tateno F, Kishi M, Tsuyusaki Y, Terada H, Inaoka T. MIBG myocardial scintigraphy in pre-motor Parkinson's disease: a review. Parkinsonism Relat Disord. 2014;20:267-273.

9. Tsujikawa K, Hasegawa Y, Yokoi S, et al. Chronological changes of ${ }^{123}$ I-MIBG myocardial scintigraphy and clinical features of Parkinson's disease. J Neurol Neurosurg Psychiatry. 2015;86:945-951.

10. Kim JS, Park HE, Park IS, et al. Normal 'heart' in Parkinson's disease: is this a distinct clinical phenotype? Eur J Neurol. 2017;24:349-356.

11. Miyazaki Y, Sako W, Sato K, Izumi Y, Kaji R. MIBG myocardial scintigraphy can predict the occurrence of wearing-off phenomenon in early-stage Parkinson's disease. J Neurol Disord. 2014;2:100154.

12. Gibb WR, Lees AJ. The relevance of the Lewy body to the pathogenesis of idiopathic Parkinson's disease. J Neurol Neurosurg Psychiatry. 1988;51:745-752.

13. Stacy M, Bowron A, Guttman M, et al. Identification of motor and nonmotor wearing-off in Parkinson's disease: comparison of a patient questionnaire versus a clinician assessment. Mov Disord. 2005;20:726-733.
14. Tomlinson CL, Stowe R, Patel S, Rick C, Gray R, Clarke CE. Systematic review of levodopa dose equivalency reporting in Parkinson's disease. Mov Disord. 2010;25:2649-2653.

15. Agostini D, Carrio I, Verberne HJ. How to use myocardial ${ }^{123}$ I-MIBG scintigraphy in chronic heart failure. Eur J Nucl Med Mol Imaging. 2009;36:555-559.

16. Stocchi F. The levodopa wearing- off phenomenon in Parkinson's disease: pharmacokinetic considerations. Expert Opin Pharmacother. 2006;7:1399-1407.

17. Engelender S, Isacson O. The threshold theory for Parkinson's disease. Trends Neurosci. 2017;40:4-14.

18. Gross M, Bannister R, Godwin-Austen R. Orthostatic hypotension in Parkinson's disease. Lancet. 1972;1:174-176.

19. Hallett PJ, McLean JR, Kartunen A, Langston JW, Isacson O. $\alpha$-Synuclein overexpressing transgenic mice show internal organ pathology and autonomic deficits. Neurobiol Dis. 2012;47:258-267.

20. Kuo YM, Li Z, Jiao Y, et al. Extensive enteric nervous system abnormalities in mice transgenic for artificial chromosomes containing Parkinson disease-associated alpha-synuclein gene mutations precede central nervous system changes. Hum Mol Genet. 2010;19:1633-1650.

21. Ahlskog JE, Muenter MD. Frequency of levodopa-related dyskinesias and motor fluctuations as estimated from the cumulative literature. Mov Disord. 2001;16:448-458.

22. Stocchi F, Antonini A, Barone P, et al. Early detection of wearing off in Parkinson disease: the DEEP study. Parkinsonism Relat Disord. 2014;20:204-211.

23. Yoritaka A, Shimo Y, Takanashi M, et al. Motor and non-motor symptoms of 1453 patients with Parkinson's disease: prevalence and risks. Parkinsonism Relat Disord. 2013;19:725-731.

24. Sato K, Hatano T, Yamashiro K, et al. Prognosis of Parkinson's disease: time to stage III, IV, V, and to motor fluctuations. Mov Disord. 2006;21:1384-1395.

25. Warren Olanow C, Kieburtz K, Rascol O, et al. Factors predictive of the development of levodopa-induced dyskinesia and wearing-off in Parkinson's disease. Mov Disord. 2013;28:1064-1071.

26. Kostic V, Przedborski S, Flaster E, Sternic N. Early development of levodopainduced dyskinesias and response fluctuations in young-onset Parkinson's disease. Neurology. 1991;41:202-205.

27. Schrag A, Hovris A, Morley D, Quinn N, Jahanshahi M. Young- versus olderonset Parkinson's disease: impact of disease and psychosocial consequences. Mov Disord. 2003;18:1250-1256.

28. Jankovic J. Motor fluctuations and dyskinesias in Parkinson's disease: clinical manifestations. Mov Disord. 2005;20:S11-S16.

29. Colombo D, Abbruzzese G, Antonini A, et al. The "gender factor" in wearingoff among patients with Parkinson's disease: a post hoc analysis of DEEP study. ScientificWorldJournal. 2015;2015:787451.

30. Chen H, Fang J, Li F, Gao L, Feng T. Risk factors and safe dosage of levodopa for wearing-off phenomenon in Chinese patients with Parkinson's disease. Neurol Sci. 2015;36:1217-1223.

31. Fukae J, Ishikawa K, Hatano T, et al. Serum uric acid concentration is linked to wearing-off fluctuation in Japanese Parkinson's disease patients. J Parkinsons Dis. 2014;4:499-505. 